\title{
Strengthening Post-Operative Pain Assessment in Patients with Major Abdominal Surgery, University Teaching Hospitals, Zambia
}

\author{
Ruth Wahila1 ${ }^{*}$, Etienne Bwana-Fwamba-Koshe Odimba², Catherine Ngoma ${ }^{3}$ \\ ${ }^{1}$ Department of Basic and Clinical Nursing Sciences, School of Nursing Sciences, University of Zambia, Lusaka, Zambia \\ ${ }^{2}$ Department of Surgery, School of Medicine, University of Zambia, Lusaka, Zambia \\ ${ }^{3}$ Department of Midwifery, Women's and Child Health, School of Nursing Sciences, University of Zambia, Lusaka, Zambia \\ Email: ^ruthwahila@yahoo.co.uk
}

How to cite this paper: Wahila, R., Odimba, E.B.-F.-K. and Ngoma, C. (2020) Strengthening Post-Operative Pain Assessment in Patients with Major Abdominal Surgery, University Teaching Hospitals, Zambia. Pain Studies and Treatment, 8, 23-34.

https://doi.org/10.4236/pst.2020.82002

Received: March 6, 2020

Accepted: April 17, 2020

Published: April 20, 2020

Copyright $\odot 2020$ by author(s) and Scientific Research Publishing Inc. This work is licensed under the Creative Commons Attribution International License (CC BY 4.0).

http://creativecommons.org/licenses/by/4.0/

\section{(c) (i) Open Access}

\begin{abstract}
Systematic, routine pain assessment using standardized clinical guidelines is the foundation of effective pain management for patients who are unable to self-report pain. In Zambia, there are no context appropriate standardised clinical guidelines for post-operative pain observations. This study sought to develop such a clinical guideline in form of an assessment tool. The study adopted an exploratory sequential mixed method through a three-phased approach and an adapted Clinical Decision Making Survey instrument was used. Snowball sampling was employed and in phases II and III, purposive sampling was used. The study was conducted at the University Teaching Hospitals where 120 participants were enrolled in the study. Phases II and III provided preliminary internal validation processes of the developed tool, where discussions, orientation and trial implementation of the tool were done. In phase II of the study, 47 participants comprising of nurses participated while in phase III, there were 11 nurses and 32 participants. The results yielded the first ever standardised post-operative pain assessment tool for patients with major abdominal surgery in Zambia. The tool is made up of six dimensions of the identified nonverbal indicators of post-operative pain in patients with major abdominal surgery namely: facial expressions, mobility, activity intolerance, behavioural disturbance, communication ability and vital signs. The present study showed that the developed post-operative pain assessment tool for Zambia is acceptable for use among patients who have had major abdominal surgery and can facilitate improved post-operative pain management for most patients.
\end{abstract}

\section{Keywords}

Development, Post-Operative Pain, Assessment Tool, Nonverbal Indicators, 
Major Abdominal Surgery

\section{Introduction}

Reported worldwide, the level of post-operative pain varies from $14 \%$ to $70 \%$ depending on the intensity considered, type of surgery and anaesthesia, time of data collection, and institutional protocol for pain management [1]. According to one of the pain characteristics, if the rate of tissue injury (extent of damage per unit time) is high, the intensity of pain is also high and this could be said to explain the high rate of post-operative pain among patients with major abdominal surgeries. The abdominal wall is one of the largest organs of the body that protects many internal organs and thus, major abdominal surgeries tend to cover a wide surface area in an attempt to ensure that an anatomical and physiological alteration in one organ has not affected other organs. It is therefore predictable that the levels of post-operative pain among patients will be high.

The level of moderate to severe pain among patients following abdominal surgery is about $70 \%$ [2]. In some patients, the levels of post-operative pain experienced by patients are as high as $84.17 \%, 92.5 \%$ and $96.66 \%$ at the fifth post-operative hour, second and third post-operative day, respectively [3]. In addition, it has been repeatedly confirmed by studies in the past 3 to 4 decades that $20 \%$ to $80 \%$ of patients who had undergone surgery suffer from inadequately treated pain [4] [5] due to an interaction of many factors including biological, psychological, environmental, and social factors [6] which influence pain assessment. The high percentage of the levels of post-operative pain experienced by patients has huge negative implications for the patients and the health care system. Just like in all pain conditions, one of the essential elements to provide optimal relief for these patients with major abdominal surgery suffering from extreme levels of post-operative pain is through pain assessment. The first step in providing adequate pain relief for patients with major abdominal surgery is appropriate assessment [7].

Patients' self-reports of pain should be obtained because these reports are the most valid measure of pain [8]. Unfortunately, especially in surgical units, many factors may alter verbal communication with patients, such as administration of sedative agents [8]. Of particular significance to countries like Zambia, anecdotal data reveal that one of the critical hindrances to the use of self-reports as the gold standard of post-operative pain assessment is culture and age [9]. Different considerations arise regarding cultural validity when considering self-report issues and observational/judgment issues. Unlike pain arising from the disease state, post-operative pain arises from the surgical intervention. At most times, many of these post-surgical patients in Zambia are of the view that it is natural to experience post-operative pain while others seem to feel that verbalisation of the pain experience is a sign of being weak. Such feelings, which arise from the 
socialisation process of individuals, may hinder the "factual" expression of self-reports of post-operative pain.

When patients cannot express themselves in any way, observable indicators, clustered into physiological and behavioural categories, become unique indices for the assessment of pain. Thus, many post-operative patients are likely to use facial expressions or hand motions or by seeking attention with other movements to denote pain. It was therefore critical that the first ever standardised post-operative pain assessment tool for Zambia that considers the post-surgical patients' cultural system be developed in order to provide quality pain assessment.

Effective pain management has become an integral part of modern surgical practice and the nursing profession. Hence, an aggressive pain management protocol that incorporates use of pain assessment tools as one of the methods for assessing post-operative pain is required for consequent effective pain relief. Such an approach has positive benefits for the patient's quality of life following surgery, accessibility to surgical interventions and reduced costs for the health care system especially for Zambia which is on the trajectory of achieving middle economic status by the year 2030 [10]. Patients who have experienced inadequate pain management may also be reluctant to seek medical care for other health problems. Hence, effective management of post-operative pain not only reduces patient suffering but also reduces morbidity which facilitates rapid recovery and early discharge from hospital, leading to reduced hospital costs.

It is for this reason that the cornerstone of optimal pain management must take into account all the factors that have an effect on pain assessment. Though the patient's self-report of pain is considered to be the most reliable measure, other methods of pain assessment must be incorporated to allow for an all-inclusive evaluation of post-operative pain [7]. Pain assessment tools could also be utilised as invaluable guides for assessing post-operative pain. It is against this backdrop that this study focused on developing a post-operative pain assessment tool for Zambia for use among patients who have had major abdominal surgery.

\section{Methodological Approaches}

\subsection{Design, Sample and Setting}

This study based on the pragmatic paradigm used an exploratory sequential mixed methods research design. Through a three-phased approach, the nonverbal indicators of post-operative pain in patients who have had major abdominal surgery were explored by heralding triangulation of multiple data sources as a means of converging, connecting and embedding the truth about post-operative pain and its assessment measures.

In phase I of the study, 30 key informants comprising of surgeons and nurses were identified through snowball sampling method and interviewed. Phase II of the study included 47 participants, all of whom were nurses working from the 
surgical units. The nurses provided an initial internal validation of the post-operative pain assessment tool for Zambia. In phase III, there were two groups of participants. The first group of participants was 11 nurses who conducted a trial implementation of the post-operative pain assessment tool for Zambia and providing a second internal validation of the tool. The second group of participants was 32 post-operative patients with major abdominal surgery on whom the post-operative pain assessment tool for Zambia was implemented on. All the participants in phases II and III of the study were purposively selected.

The criteria for inclusion in the study were as follows:

1) Registration with the professional regulatory body;

2) In phase I, nurses and surgeons who had consecutively worked on the surgical wards of UTH for more than three months and were actively involved in providing direct care to patients who had undergone major abdominal surgery;

3) In phase II, all the nurse managers at UTH as well as nurses from the all the surgical units of the facility;

4) During phase III, nurses practicing on the general surgical units of UTH for a period of more than three months and were involved in providing direct patient care to patients who have had major abdominal surgeries;

5) All participants who were willing to participate in the study.

Only participants who were not present at the time of the study were excluded from participation.

The study was conducted in the general surgical wards of the University Teaching Hospitals (UTH). UTH is the largest health institution and main specialist referral centre from across Zambia and thus caters for a very diverse multicultural local post-operative pain population. The burden of surgical conditions and diseases is increasing in low-income and middle-income countries, but the capacity to meet the demands they present is not following pace (Chisoso, 2012) and UTH, being the main specialised health facility has a high turnover of surgical patients. The diverse and large volume of post-surgical patients is thought to expose nurses to the complexity of post-operative pain that the patients may experience and for which nurses provide care. The use of a post-operative pain assessment tool for Zambia may thus facilitate reduced hospital stay for patients and other challenges faced by clinicians such as overwhelming work and post-operative pain complexity.

During the research process, qualitative data was first collected and analyzed, and the identified post-operative pain indicators were used to drive the development of a quantitative instrument (post-operative pain assessment tool) to further explore the research concept [11] [12] [13]. As a result, three stages of analyses were conducted: after the primary qualitative phase, after the secondary quantitative phase, and at the post implementation after a trial implementation of the post-operative pain assessment tool for Zambia.

\subsection{Instrument and Procedure}

The instrument used in this study was adapted from the Clinical Decision Mak- 
ing Survey tool for pain management [14]. During each phase of the study, the instrument was modified to suit the information required from the participants.

The content and design of the schedule is clear and simple, but comprehensive enough to categorically provide guidance for pain assessment from a wide variety of factors such as perception of nurses towards pain intensity experienced by post-operative patients. Beyond ease of administration, an additional advantage of the clinical decision making tool for pain management survey may be its utility as a tool for education. In addition, the instrument provided rapid depiction of the greatest influences for pain assessment within individual patients and suggested strategies for management of pain.

\subsection{Data Analysis}

Due to the epidemiological inquiry type of the study, data were analysed using descriptive statistics. The percentage of findings was calculated on the basis of the total number of participants who answered each question. All the participants who took part in the study were included in the analysis.

\section{Results}

Table 1 shows the socio-demographic characteristics of the participants through the three phases of the study.

Table 1. Socio-demographic characteristics of participants.

\begin{tabular}{|c|c|c|c|c|}
\hline \multirow{2}{*}{\multicolumn{2}{|c|}{ Characteristic }} & \multicolumn{3}{|c|}{ Percentage } \\
\hline & & \multirow{2}{*}{$\begin{array}{c}\text { Phase } 1 \\
33.3\end{array}$} & \multirow{2}{*}{$\begin{array}{c}\text { Phase } 2 \\
80.9\end{array}$} & \multirow{2}{*}{$\begin{array}{c}\text { Phase } 3 \\
72.7\end{array}$} \\
\hline Gendler & Male & & & \\
\hline teres & Female & 66.7 & 19.1 & 27.3 \\
\hline & $21-25$ & 6.7 & 4.3 & - \\
\hline & $26-30$ & 11.0 & 55.2 & - \\
\hline & $31-35$ & 16.7 & 23.5 & - \\
\hline \multirow[t]{5}{*}{ Age } & $36-40$ & 16.7 & 10.7 & - \\
\hline & $41-45$ & 13.3 & 6.4 & - \\
\hline & $46-50$ & 16.7 & - & - \\
\hline & $\leq 51$ & 11.0 & - & - \\
\hline & Certificate & 3.3 & 4.3 & - \\
\hline \multirow[t]{5}{*}{ Educational Level } & Diploma & 90.0 & 93.6 & 90.9 \\
\hline & Degree & 6.7 & 2.1 & 9.1 \\
\hline & $>1$ Year & 10.0 & 6.4 & 9.1 \\
\hline & $1-5$ Years & 20.0 & 57.5 & 72.8 \\
\hline & 6 - 10 Years & 16.7 & 29.7 & 9.1 \\
\hline \multirow[t]{4}{*}{ Work Experience } & $11-15$ Years & 6.7 & 4.2 & 9.1 \\
\hline & 16 - 20 Years & 26.7 & 2.1 & - \\
\hline & $21-25$ Years & 13.3 & - & - \\
\hline & $\geq 26$ Years & 6.7 & - & - \\
\hline
\end{tabular}


Phase I of the study revealed that $93.3 \%$ of the participants reported that they assessed the post-operative pain through abnormal facial expressions depicting discomfort. Failure to mobilise normally was also reported as a nonverbal indicator of post-operative pain according to $90 \%$ of the participants. Further, $80 \%$ of the participants mentioned alteration in normal pattern of behaviour in the form of restlessness, sleeplessness, crying, groaning, tearing, irritable conduct, refusal to eat and concentration difficulties. A considerable percentage of participants (76.7\%) also cited activity intolerance as a nonverbal indicator of post-operative pain. Only slightly more than one-third (36.7\%) of the participants indicated that changes in vital signs in the form of increased parameters including sweating could be used as post-operative pain nonverbal indicators. A percentage of $40 \%$ of the participants cited verbalisation of post-operative pain signifying the ability of patients to self-report pain.

Phase II results show that participants recommended that clinicians should mainly assess the patient's mobility state, facial expressions and the patient's state of general behaviour to observe post-operative pain. Using a scale of $0 \%$ to $100 \%$, findings revealed that $100 \%$ of the participants were assessing patients' abnormal facial expressions, $91.5 \%$ assessed the patients' level of immobility and $70.2 \%$ assessed the patients' change in general behaviour to determine whether the patient was experiencing pain following major abdominal surgery. Further findings revealed that $57.4 \%$ assessed the patients' change in vital signs while activity intolerance was only suggested by 18 (38.3\%) of the participants. The study results brought forth difficult communication as an indicator of postoperative pain following major abdominal surgery although the percentage of the participants stating likewise was just slightly more than a quarter (31.9\%).

The results for phase III are summarised in Table 2.

Table 2. Non-verbal post-operative pain indicators observed in phase III of Study.

\begin{tabular}{|c|c|c|c|c|}
\hline \multicolumn{3}{|c|}{ POP Indicator } & Frequency & Percentage \\
\hline \multicolumn{5}{|c|}{ Abnormal Facial Expressions } \\
\hline & \multicolumn{2}{|l|}{ Yes } & 9 & 81.8 \\
\hline & \multicolumn{2}{|l|}{ No } & 2 & 18.2 \\
\hline \multicolumn{5}{|l|}{ Difficult mobilization } \\
\hline & \multicolumn{2}{|l|}{ Yes } & 7 & 63.6 \\
\hline & \multicolumn{2}{|l|}{ No } & 4 & 36.4 \\
\hline \multicolumn{5}{|c|}{ Patient's Inability to Comply to Activity (Activity intolerance) } \\
\hline & \multicolumn{2}{|l|}{ Yes } & 7 & 63.6 \\
\hline & \multicolumn{2}{|l|}{ No } & 4 & 36.4 \\
\hline \multicolumn{5}{|l|}{ Vital Signs Changes } \\
\hline & \multicolumn{2}{|l|}{ Yes } & 19 Responses & 57.6 \\
\hline & \multicolumn{2}{|l|}{ No } & 14 Responses & 42.4 \\
\hline \multirow[t]{2}{*}{ - Increased Heart Rate } & & Yes & 10 & 90.9 \\
\hline & & No & 1 & 9.1 \\
\hline
\end{tabular}




\section{Continued}

- Increased Blood Pressure

- Fever
Yes

No

Yes

Yes

No
6

5

3

8

20 Responses

13 Responses

\section{9}

- Sleeplessness

- Lack of Concentration/Hallucinations/ Confusion

- Restlessness (Irritation or Discomfort)

Difficult Communicating (Crying/Tearing or Complaining)

Yes

Verbalising of Pain (Self-report)

\begin{tabular}{lll} 
Yes & 3 & 27.3 \\
No & 8 & 72.7 \\
\hline
\end{tabular}

\section{Discussion}

The current study resulted into the development of the post-operative pain assessment tool for Zambia which consists of six measurable indicators that could be used to observe post-operative pain among patients with major abdominal surgery. Patient mobility, level of tolerance to activity, facial expression, change in vital signs, behavioural disturbances and communication difficulty have been identified as categories of potential pain indicators in post-surgical with major abdominal surgery. An explanation to these findings is the symbolization of the effects of post-operative pain in all body systems. In fact, some authors have suggested that nonverbal behaviour may be more meaningful and easier to use in assessing pain than verbal communication [15] [16] [17] [18]. It has been recommended that in patients who are unable to adequately express their pain or the level of pain verbally, in written form, or using the Wong-Baker FACES Pain Rating Scale, nurses could know if the patient is in pain by using many of the nonverbal indicators of pain [18]. Some of the nonverbal indicators of pain that were cited in the report are facial expressions, vocal nonverbal complaints, limited verbal complaints, bracing, behavioural disturbances, restless, easy tiredness, sleeplessness, refusal to eat, nausea, non-compliant, silence, sweating, fever and agitation; and are similar to those identified in this study. However, in this study, the identified nonverbal indicators have been summarised into six by 
grouping related responses to allow for a user friendly tool.

According to the study results shown in Figure 1, there was a relative agreement among all the participants throughout the three phases on the use of facial expressions as a nonverbal indicator of post-operative pain in patients who have undergone major surgery of the abdomen. In phase I, approximately $93 \%$ of the participants recommended the use of negative changes in facial expressions as a nonverbal indicator of post-operative pain. In phase II, the percentage of participants agreeing to facial expressions as a nonverbal indicator of post-operative pain increased to $100 \%$ but reduced moderately to about $82 \%$ in phase III. Despite the decrease noted in phase III in the percentage of participants recommending facial expressions as a post-operative pain indicator, the proportion of $80 \%$ is still a high. Facial expressions are the most commonly used indicators in response to acute procedural pain in the clinical and research realm due to their objectivity, universality, accessibility, sensitivity, and specificity [19].

The second nonverbal indicator of post-operative pain that has been identified is patient mobility. This study revealed that there was no significant difference in the ratings of mobility as a nonverbal indicator of post-operative pain provided by participants in phases I and II. A total of $90 \%$ and approximately $92 \%$ of the participants in phases I and II respectively reported that observing mobility changes among patients with major abdominal surgery is a good measure of post-operative pain. Mobility assessment can help identify and encourage the use of appropriate treatment modalities that are likely to reduce the effects of immobility which are traumatising to the patient in all the facets of health; namely, physical, social and psychological wellbeing [20]. Since patient care in hospitals is bed-centric, immobility due to the presence of pain among surgical patients may make them lose muscle mass, become deconditioned to reality very quickly and worse still, suffer worse physiological trauma owing to the disruption in the integrity of the skin [21]. Pain assessment thus underscores the importance

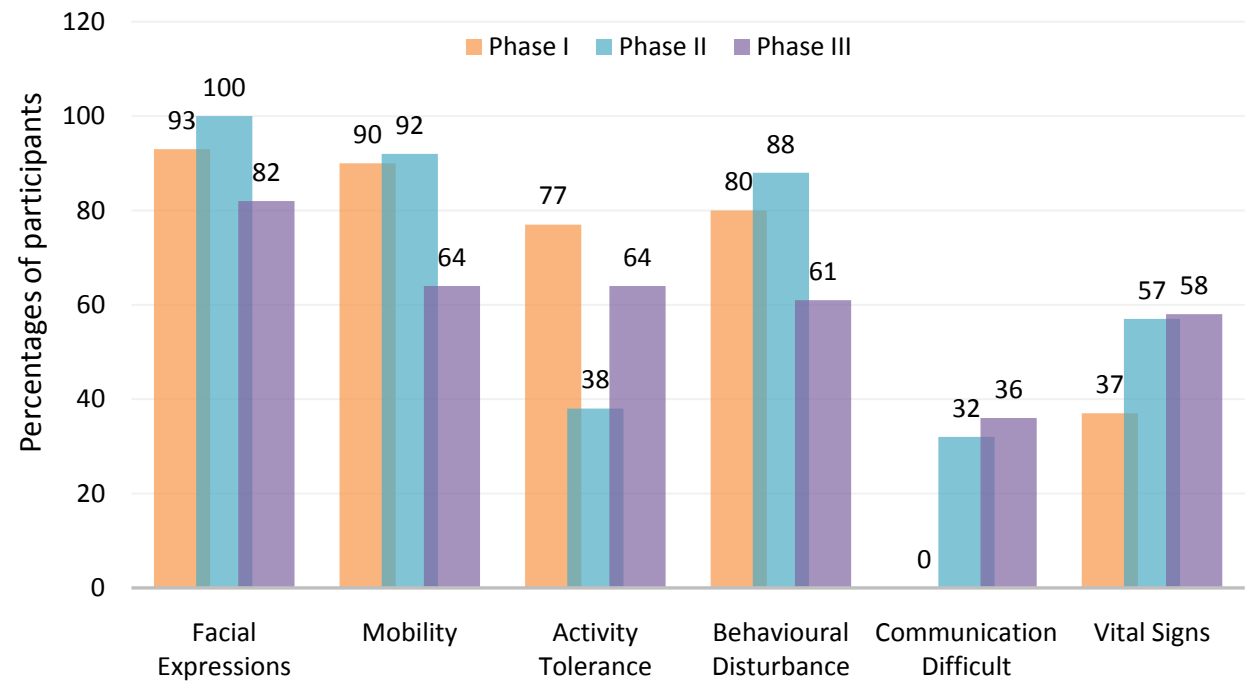

Figure 1. Nonverbal post-operative pain indicators in patients with major abdominal surgery. 
of incorporating mobility status to help avoid such physical, social and psychological impacts of pain [22]

The third nonverbal indicator of post-operative pain identified is the level of tolerance to activity. The study revealed that $77 \%$ of participants in phase I, $38 \%$ in phase II and $64 \%$ of the participants in phase III observed that level of patient tolerance to activity was a key aspect for inclusion into the post-operative pain assessment tool for Zambia as a way of evaluating patient behaviour to post-operative pain. Participants noted that activity intolerance can be measured by whether or not the patient is able to initiate and sustain an activity or not and to what extent that particular patient is able to do so. There was a significant consensus on the extent of the ability to initiate and sustain an activity with ease, to a lesser extent, failure to sustain an activity immediately and total failure to initiate and sustain an activity. Activity tolerance testing is an important diagnostic and prognostic tool for assessing pain among patients with different ailments or situations. Activity tolerance testing (also known as exercise tolerance testing or simply exercise testing or exercise stress testing) is used routinely in evaluating patients who present with chest pain, in patients who have chest pain on exertion, and in patients with known ischaemic heart disease. The test has a sensitivity of $78 \%$ and a specificity of $70 \%$ for detecting coronary artery disease [23].

Behavioural disturbance is the fourth nonverbal post-operative pain indicator that has been identified. In phase I, approximately $80 \%$ of participants reported that noting any behavioural disturbances among post-surgical patients who have had major abdominal surgery would be helpful in detecting pain levels. In phase II, $88 \%$ of the participants reported likewise and in phase III, the results were similar although the percentage of participants who indicated so fairly reduced to $64 \%$. In individuals who are unable to self-report pain due to various reasons, pain indicators may present as behaviours that are characteristic of other unmet needs [24]. The pain behaviour presents the way in which someone, in this case the clinician, can see that the other person (patient) is in pain.

The fifth behavioural nonverbal indicator of post-operative pain that was identified was communication difficult. The study though reveals that none of the participants in phase I reported communication difficult as a nonverbal indicator of post-operative pain in patients with major abdominal surgery. In phases II and III, $32 \%$ and $36 \%$ of the participants respectively cited communication difficult as an indicator of post-operative pain. Though the percentages of participants indicating communication difficult as a nonverbal indicator of post-operative pain was below $50 \%$, this indicator has been added to the post-operative pain assessment tool for Zambia following a critical reflection of the mechanisms of pain and its expression. The pain experience and its appreciation by the individual is a multifaceted phenomenon that can be relayed in so many. Certain nonverbal cues may be subtle but could provide the only means to the detection of pain. It is therefore paramount that any clues to nonverbal cues of identifying post-operative pain among patients be included in the post-operative pain assessment tool for Zambia. However, there is a need to 
examine the extent of the effect of post-operative pain on patients' ability to communicate effectively so as to provide a concrete externally validated post-operative pain assessment tool for Zambia.

Several guidelines suggest that in addition to observation of behaviours, pain assessment in the critically ill should include consideration of physiological measures such as heart rate, blood pressure, and respiratory rate. These are collectively known as vital signs together with temperature and are included on the post-operative pain assessment tool for Zambia as final nonverbal indicators of post-operative pain. In phase I, about $37 \%$ of the participants were in agreement that abnormal vital signs of pulse, respirations and blood pressure provided an indication of the existence of pain following surgery. Participants who reported vital signs as an indicator of post-operative pain in phases II and III were 57\% and 58\% respectively. Participants reported that they had observed that the vital signs usually normalised after administration of pain relief medications. Thus, participants felt that this could be one of the pain assessment measures for post-operative patients in Zambia. Since the autonomic nervous system may be activated during exposure to a painful event, fluctuations in vital signs could be indicative of the presence of pain. In one study with 30 surgical and trauma ICU participants, mean arterial pressure (MAP) and heart rate (HR) were found to increase significant during nociceptive procedures (turning and endotracheal suctioning) compared to nonnociceptive procedures (compression stocking applications and catheter dressing change) [25].

According to the health care policy and research report of 2015, it was documented that it was important that patients reported and provided information about pain and this was considered as the most valid measure [8]. However, due to a number of limitations resulting from many factors that altered the verbal communication, many clinicians were advised to use facial expressions or hand motions or by seeking attention with other movements to denote pain. Similarly, when patients cannot express themselves in any way, observable indicators, clustered into physiological and behavioral categories, become unique indices for the assessment of pain [26].

\section{Conclusion}

Today's approach to pain after surgery takes into account patient differences in the experience and report of pain, preferences among possible treatments, and response to therapy; reflecting factors such as gender and ethnicity. The approach also recognizes (in some countries) that management of post-operative pain has become a medical subspecialty owing to the growth of knowledge and specialized techniques. The integration of pain assessment tools will therefore strengthen the pain observation methods for patients and thus help relieve their untold suffering.

\section{Ethical Approval}

This study was approved by the University of Zambia Biomedical Research Eth- 
ics Committee (UNZABREC).

\section{Conflicts of Interest}

The authors declare no conflicts of interest regarding the publication of this paper.

\section{References}

[1] Bernhofer, E.I. and Sorrell, J.M. (2014) Nurses Managing Patients' Pain May Experience Moral Distress. Clinical Nursing Research, 24, 401-414.

https://doi.org/10.1177/1054773814533124

[2] Kalolo, M.D. (2011) Doctors and Nurses' Knowledge and Use of Pain Assessment Tools: A Case Study of Two Tertiary Hospitals, Lusaka.

[3] Prashant, K.S., Saikia, P. and Lahakar, M. (2016) Prevalence of Acute Post-Operative Pain in Patients in Adult Age-Group Undergoing Inpatient Abdominal Surgery and Correlation of Intensity of Pain and Satisfaction with Analgesic Management: A Cross-Sectional Single Institute-Based Study. Indian Journal of Anaesthesia, 60, 737-743. https://doi.org/10.4103/0019-5049.191686

[4] Tong, J.G. (2017) Poorly Controlled Postoperative Pain: Prevalence, Consequences, and Prevention. Journal of Pain Research, 10, 2287-2298.

https://doi.org/10.2147/JPR.S144066

[5] Corbett, A., Achterberg, W., Husebo, B., Lobbezoo, F., de Vet, H., Kunz, M., Strand, L., Constantinou, M., Tudose, C., Kappesser, J., de Waal, M. and Lautenbacher, S. (2014) An International Road Map to Improve Pain Assessment in People with Impaired Cognition: The Development of the Pain Assessment in Impaired Cognition (PAIC) Meta-Tool. BMC Neurology, 14, Article No. 229. https://doi.org/10.1186/s12883-014-0229-5

[6] Australian and New Zealand College of Anaesthetists and Faculty of Pain Medicine (2005) Acute Pain Management: Scientific Evidence. 2nd Edition, 14-16.

[7] Wahila, R., Odimba, E. and Ngoma, C. (2018) Developing a Pain Assessment Tool for Patients after Major Abdominal Surgery. British Journal of Nursing, 27, 503-507. https://doi.org/10.12968/bjon.2018.27.9.503

[8] International Association for the Study of Pain (2018) Health Care Policy and Research. Washington DC.

[9] University Teaching Hospitals, Zambia (2015) The 2015 Quarterly Facility Preliminary Report. Lusaka.

[10] Ministry of Health (2017) National Surgical, Obstetric, and Anaesthesia Strategic Plan (NSOASP), Year 2017-2021, Republic of Zambia, Lusaka.

[11] Creswell, J.W. and Plano Clark, V.L. (2011) Designing and Conducting Mixed Methods Research. 2nd Edition, SAGE Publications, Thousand Oaks.

[12] Teddlie, C. and Abbas, T. (2008) Foundations of Mixed Methods Research: Integrating Quantitative and Qualitative Techniques in the Social and Behavioural Sciences. SAGE Publications, Thousand Oaks.

[13] Onwuegbuzie, A.J., Bustamante, R.M. and Nelson, J.A. (2010) Mixed Research as a Tool for Developing Quantitative Instruments. Journal of Mixed Methods Research, 4, 56-78. https://doi.org/10.1177/1558689809355805

[14] Ferrell, B.R., Eberts, M.T., McCaffery, M. and Grant, M. (2012) The Clinical Decision Making Survey (CDMS) Measurement Instrument Database for the Social 
Science. https://www.midss.org/sites/default/files/clinical_decision_making.pdf

[15] Francis, L. and Fitzpatrick, J.J. (2013) Postoperative Pain: Nurses' Knowledge and Patients' Experiences. Pain Management Nursing, 14, 351-357. https://doi.org/10.1016/j.pmn.2012.05.002

[16] Pasero, C. and McCaffery, M. (2011) Pain Assessment and Pharmacologic Management. Mosby-Elsevier, St. Louis.

[17] Breivik, H. and Stubhaug, A. (2010) Management of Acute Postoperative Pain: Still a Long Way to Go! Pain, 137, 233-234. https://doi.org/10.1016/j.pain.2008.04.014

[18] Herr, K. (2011) Pain Assessment Strategies in Older Patients. The Journal of Pain, 12, S3-S13. https://doi.org/10.1016/j.jpain.2010.11.011

[19] Desrosiers, A., Harrison, D. and Letham, A. (2015) Use of Facial Expressions for Pain Assessment in Infants during Acute Painful Procedures. Pediatric Pain Letter, 17, 5-8.

[20] Schroeder, L.D., Hoffman, L.A., Fioravanti, M., Medley, P.D., Zullo, G.T. and Tuite, K.P. (2016) Enhancing Nurses' Pain Assessment to Improve Patient Satisfaction. Orthopaedic Nursing, 35, 108-117.

[21] Dickinson, S., Tschannen, D. and Shever, L.L. (2013) Can the Use of an Early Mobility Program Reduce the Incidence of Pressure Ulcers in a Surgical Critical Care Unit? Critical Care Nursing Quarterly, 36, 127-140. https://doi.org/10.1097/CNQ.0b013e31827538a1

[22] Moyle, S. (2015) Pain Assessment and Management.

[23] Hill, J. and Timmis, A. (2003) Exercise Tolerance Testing. In: Morris, F., Edhouse, J., Brady, W.J. and Camm, J., Eds., (2008) ABC of Clinical Electrocardiography, BMJ Publishing Group, London, 41-45.

[24] Roghieh, N., Yaghoobzadeh, A., et al. (2018) Behavioural Pain Indicators in Patients with Traumatic Brain Injury Admitted to an Intensive Care Unit. Journal of Caring Sciences, 7, 197-203. http://journals.tbzmed.ac.ir/JCS https://doi.org/10.15171/jcs.2018.030

[25] Payen, J.-F., Bru, O. and Bosson, J.-L. (2011) Assessing Pain in Critically Ill Sedated Patients by Using a Behavioral Pain Scale. Critical Care Medicine, 29, 2258-2263. https://doi.org/10.1097/00003246-200112000-00004

[26] Odai, E.D., Ehizele, A.O. and Enabulele, J.E. (2015) Assessment of Pain among a Group of Nigerian Dental Patients. BMC Research Notes, 8, Article No. 251. https://doi.org/10.1186/s13104-015-1226-5 